\title{
Experimental Evaluation of a Heat Transport System for a High- Temperature Storage Unit
}

\author{
Tim Lanz, Werner Kraft, Veronika Stahl, Peter Vetter, Benedikt Sebastian Volk \\ ${ }^{1}$ Institute of Vehicle Concepts/German Aerospace Center (DLR) \\ Pfaffenwaldring 38-40, 70569 Stuttgart, Germany \\ tim.lanz@dlr.de; werner.kraft@dlr.de; veronika.stahl@dlr.de; peter.vetter@dlr.de; benedikt.volk@dlr.de
}

\section{Extended Abstract}

Future road vehicles will have partially or fully electrified drive concepts. In comparison with conventionally powered vehicles, there is comparatively little waste heat available for air conditioning and temperature regulation purposes. This lack of waste heat with simultaneously increasing demands on efficiency and comfort, places great demands on future thermal management systems. It is particularly evident in the thermal management of fully electrified vehicles on cold winter days. Standard PTC heaters use electrical energy from the traction battery and convert it into heat. In this process, the drive system loses this energy, which results in a range reduction of the vehicle by up to 50 per cent [1].

This challenge is targeted by heat storage systems, that provide thermal energy through comparatively small, lightweight high-performance and cost-effective systems.

The thermal storage system, developed by DLR, utilises the sensitive change in temperature and the phase change of the storage material to store energy. During charging, the metallic Phase Change Material (mPCM) is heating and melting, while during discharging, it is cooling and solidifying. Intelligent need-based charging can be carried out in parallel when charging the traction battery at the charging station. The heat released during the subsequent discharging can be made available to the interior of the car.

A possible approach to make the stored thermal energy accessible is to thermally link the storage unit with the cooling cycle of the car. Due to high temperatures, which would evaporate the coolant, a flow through the storage unit is not possible. Instead, a concept for indirect coupling was developed to transport a certain amount of heat within a closed circuit between both systems. Within this concept, evaporation and condensation of a working fluid are the dominating transport phenomena.

Numerical investigations were undertaken within the simulation software Dymola to estimate the performance of the developed concept. As a further step, the validation of the numerical analysis was targeted by an experimental investigation. Therefore, a test bench was built and experimental series were undertaken.

A methodical approach for developing and assembling this test bench within the defined objectives will be presented. Therefore, the requirements and boundary conditions defined by the preliminary steps will be regarded. Together with the functional analysis of the system, the foundation for developing the test bench is given and highlighted. The resulting solution principles for the sub-functions lead to the finalization of the individual components within the test bench.

The second focus of the presentation lies on the testing process and the measured quantities. Therefore, the experimental procedure will be presented, highlighting the physical variables influencing the heat transfer process. In addition, the underlying physical phenomena will be regarded and discussed concerning the functional implementation of the developed design.

Overall, the results prove the functionality of the developed concept. A maximum heat flux at approximately $5.5 \mathrm{~kW}$ between the heat source (storage unit) and the heat sink (cooling cycle) was observed. Accordingly, the targeted quantity of at least $5 \mathrm{~kW}$ was met at various design points.

Heat output larger than $5 \mathrm{~kW}$ was first met at mass flow rates of $14 \mathrm{~kg} / \mathrm{h}$ at temperatures in between 160 and $200{ }^{\circ} \mathrm{C}$. An increase of the flow rate to $16 \mathrm{~kg} / \mathrm{h}$ did not change the performance, whereas a decrease of the mass flow rate below $12 \mathrm{~kg} / \mathrm{h}$ leads to an immediate drop of the thermal output below $500 \mathrm{~W}$. This non-linear behaviour assumes that the fluid flow pattern within the evaporator is a crucial design parameter and has to be adapted in future design variations.

Comparing the experimental results with the preceding simulation, deviations of the thermal output were observed especially at low mass flow rates. This assumes that the geometrical adaption of the concept within the simulation was not appropriate and has to be revised for better accordance. 
Furthermore, optimization of the current concept is required at the current concept of the evaporator to allow more effective and adaptive heat extraction based on the system requirements. As the experiment has proven that the extraction is not ideal already. Therefore, the fluid flow and heat absorption within the evaporator has to be further investigated.

\section{References}

[1] H. Großmann, PKW-Klimatisierung - Physikalische Grundlagen und technische Umsetzung. Berlin Heidelberg: Springer, 2013 